Laboratory diagnosis in Britain of brucella infection in man unfortunately depends almost wholly on serology. Blood cultures usually yield a disappointingly low proportion of positive results, though Payne 7 reported success from over half in the acute stage. The reasons for generally poor results deserve close examination. The prevalent British biotypes may be more difficult to grow than those found in other parts of the world, or there may be other explanations. Attempts to refine serological methods ${ }^{8}{ }^{9}$ are, indeed, being pursued with great devotion. They have tended, on the whole, to be of greater academic than practical interest and-in terms of clinical diagnosis and control of treatment-not to justify the hopes often aroused by increasingly complex methods. The standard agglutination test, properly carried out with good, reliable antigen, will usually provide very little less relevant information than the numerous other tests brought in during the past 15 years. More important, the interpretation of serological results requires understanding of what they establish and what they do not. Serological evidence of infection (particularly among farmers and veterinarians) may be shown to be at a very much higher level than the incidence of clinical disability. Clinical history and signs, critically assessed, are a necessary part of any decision about whether positive serological findings, by any test, denote active disease.

1 Scottish Brucellosis Symposium, 1976, Scottish Medical fournal, 1976, 21, 123.

${ }^{2}$ Brown, A C L, Scottish Medical fournal, 1976, 21, 124.

${ }^{3}$ Madden, E, Scottish Medical fournal, 1976, 21, 135.

${ }^{4}$ Reid, D, Scottish Medical fournal, 1976, 21, 125.

5 Lancet, 1975, 1, 436.

${ }^{6}$ Henderson, R J, Lancet, 1975, 1, 585.

7 Payne, D J H, Medicine (London), 1975, No 3, 123.

${ }^{8}$ Diaz, R, Maravi-Poma, E, and Rivero, A, Bulletin of the World Health Organisation, 1976, 53, 417.

${ }^{9}$ Kerr, W R, et al, fournal of Medical Microbiology, 1968, 1, 181.

\section{Urinary tract infection in boys}

Urinary tract infection in boys is widely believed to be associated with underlying structural abnormalities of the urinary tract. Twenty years ago Macaulay and Sutton ${ }^{1}$ reassessed a group of 32 children six to seven years after their first infection at ages between 3 weeks and $4 \frac{1}{2}$ years. Five of the ten boys, all with gross abnormalities of the genitourinary tract, had died; the 22 girls were all still alive.

More recently DeLuca et $a l^{2}$ reviewed 1279 children with recurrent urinary infections. Of these, 203 (143 boys and 60 girls) had had an obstructive uropathy and in them symptoms had started early in infancy or during the first three years of life. Of the 1076 children with no evidence of obstructive uropathy, the sex ratio was reversed-834 girls and 242 boys -and their symptoms had generally started between the ages of 3 and 5 years.

Steele et $a l^{3}$ re-evaluated a group of 133 patients admitted to hospital as children with a urinary infection between 1940 and 1950. The mortality and the incidence of renal insufficiency were four times greater among the boys than the girls and were inversely related in both sexes to the age of onset. Smallpiece ${ }^{4}$ reviewed 343 children with urinary tract infections including 42 girls and 34 boys under the age of 3 months. Severe infections were common in this group; one of the boys had a urethral obstruction but the remainder had anal abnormalities with gross contamination of the urinary tract through fistulae.
Over the age of 3 months there were 219 girls and 48 boys, and renal tract abnormalities were found in $18(37 \%)$ of the boys and in $44(20 \%)$ of the girls.

Against this background it is a little surprising that two more recent studies of urinary tract infections in boys suggest that the prognosis may be less grave. Cohen's prospective study ${ }^{5}$ of 57 boys presenting with their first urinary tract infection showed that they could be divided into two groups. Boys aged under 10 years usually had fever and a high incidence $(76 \%)$ of abnormalities of the urinary tract, most commonly vesicoureteric reflux. Four boys, all aged under 10, required corrective surgery for urethral valves, ureteric reimplantation, correction of bilateral hydronephrosis and hydroureters, or ureterectomy. Boys aged 10-14 years were usually afebrile and commonly presented with frequency and dysuria. Only two out of 13 had radiological abnormalities. Moreover, in seven of the 57 boys quantitative urine cultures yielded fewer than $10^{3}$ organisms per $\mathrm{ml}$; all of these seven boys were under 10 and all had serious renal abnormalities; three required surgical correction. In the 57 boys Gram-positive cocci were more common and Escherichia coli less prevalent as the infecting organism than in a previously studied group of girls.

The second study, ${ }^{6}$ of 73 boys aged 2-12 years presenting with their first urinary tract infection, showed that 22 had radiological abnormalities - including reflux, radiological scars, duplex kidney or ureter or both, ureterocele, horseshoe kidney with hydronephrosis, obstructed megaureter and hydronephrosis, pelviureteric junction obstruction, and stone. Only two boys, however, needed urinary tract surgery. Six of the boys with radiological abnormalities had only probable or doubtful urinary infections. Proteus species predominated as the infecting organisms, and cultures of preputial swabs suggested that the source of infection in boys might be the prepuce or urethra rather than the bowel, as in girls. In that context, Bergstrom ${ }^{7}$ compared nonobstructive urinary infections in a group of 44 boys and a control group of age-matched girls presenting with their first urinary infection. Again, the boys had a higher rate of infection with Proteus species and other organisms while in the girls the predominant organism was $E$ coli. The incidence of reflux was similar, but eight out of 40 boys had radiological scarring of the kidneys compared with only one out of 30 girls.

What conclusions may we draw from these studies? Clearly we want to separate out those children, both boys and girls, who have an underlying renal abnormality, commonly some form of congenital obstructive lesion. These patients are likely to present in the early years of life, their prognosis may be poor, and surgical intervention will be necessary in some. What microbiological criteria are used for diagnosing a urinary tract infection will depend on sampling techniques. With suprapubic aspirates any bacterial growth on culture is important. Using mid-stream urine samples, $\mathrm{Kass}^{8}$ proposed that a pure growth of over $10^{5}$ organisms $/ \mathrm{ml}$ should be taken as clinically significant bacteriuria. Yet some of the patients described in the studies just reviewed had important urinary tract abnormalities and symptoms, but the results of quantitative bacterial cultures were reported as being below this level. Perhaps, therefore, we should review the criteria and include lower bacterial counts when making the diagnosis.

Children with non-obstructive urinary tract infections form a separate group. This problem is commoner in girls, and the sexes also show differences in the predominant infecting organisms. There is a considerable incidence of vesicoureteric reflux, but the very low incidence of bacteriuria in neonates ${ }^{9}$ suggests that it develops after the first month of life. 
How far, then, should urological investigation proceed in children presenting with a urinary tract infection ? Certainly it seems that urinary infections in boys are more likely to be associated with structural abnormalities of the urinary tract, but that should not lead to a rigid selective policy. Some doctors, indeed, would even claim that there is a strong case for full urological evaluation (including intravenous pyelography and micturating cystography) in any child presenting with a urinary tract infection, regardless of age or sex, but this argument would be as strongly contested by others.

1 Macaulay, D, and Sutton, R N P, Lancet, 1957, 2, 1318.

2 DeLuca, F G, Fisher, J H, and Swenson, O, New England fournal of Medicine, 1963, 268, 75.

${ }^{3}$ Steele, R E, Leadbetter, G W, Crawford, J D, New England fournal of Medicine, 1963, 269, 883.

4 Smallpeice, V, Lancet, 1966, 2, 1019.

${ }^{5}$ Cohen, M, American fournal of Diseases of Children, 1976, 130, 810.

${ }^{6}$ Hallett, R J, Pead, L, and Maskell, R, Lancet, 1976, 2, 1107.

7 Bergström, T, Archives of Disease in Childhood, 1972, 47, 227.

${ }^{8}$ Kass, E H, Transactions of the Association of American Physicians, 1956, 69, 56.

${ }^{9}$ Gower, P E, et al, Archives of Disease in Childhood, 1970, 45, 259.

\section{Diagnosis of allergy}

Hypersensitivity is an abnormal reaction to a foreign substance and is not necessarily immunological in nature. An allergic (atopic) reaction is a form of hypersensitivity that can be shown to be mediated by immune mechanisms. Clinically it is encountered most commonly as allergic rhinitis, asthma, or dermatitis. The allegedly allergic basis for many other diseases makes the precise diagnosis of allergic reactions a matter of clinical importance.

In hypersensitive individuals allergens provoke the formation of specific IgE antibody-a class of immunoglobulin that binds to basophils in the blood and mast cells in the tissues. Combination of the allergen with specific $\operatorname{IgE}$ on the cell surface leads to the release of histamine and other mediators; and, in consequence, skin testing in allergic subjects provokes a weal-and-flare reaction within 15 minutes of challenge. This sequence of events-the immediate hypersensitivity reaction -is the form of immune response that predominates in most allergic conditions. In reality, however, no immune response occurs in isolation, but the different forms are provoked to varying degree by immune stimuli. Each component of the immediate hypersensitivity reaction can be assayed in order to diagnose allergy in general terms or to implicate a particular allergen. In practice, most attention has been given to the estimation of total and antigen-specific IgE in the serum and to provocation tests. ${ }^{1}$

Total serum IgE is measured by the radioimmunoabsorbent technique (RIST), in which antibody to $\operatorname{IgE}$ is bound to Sephadex particles. The concentration of $\mathrm{IgE}$ in the test serum is assayed by its ability to displace a standardised amount of ${ }^{125}$ I-labelled IgE from combining with the antibody. A commercial kit is available for this purpose from Pharmacia. A high concentration of serum IgE points to the probability of an allergic state, making the test clinically useful, ${ }^{2}{ }^{3}$ but the procedure does not indicate which allergen is responsible. In contrast to the other immunoglobulin classes, a high proportion of the total IgE can be accounted for by its reactivity with a single allergen. ${ }^{4-6}$ Unfortunately, the test is not free from technical problems, particularly when measuring low concentrations of IgE. For example, many commercial antisera to IgE react with portions of other immunoglobulins. ${ }^{7}$ Improved methods such as double antibody radioimmunoassay ${ }^{8-10}$ circumvent these difficulties but are not yet in routine clinical use. In the past there have been conflicting reports about the concentration of IgE in the sera of patients with neoplastic and immunodeficiency diseases, but these difficulties were almost entirely methodological in origin. In contrast, reports of raised IgE concentrations in association with cot deaths, ${ }^{11}$ glomerulonephritis, ${ }^{12}$ and connective tissue diseases have been based largely on improved techniques and raise new possibilities about the pathogenesis of these conditions.

The radioallergosorbent test (RAST) is a method of detecting IgE antibody that reacts specifically with suspected allergens. ${ }^{1}$ In principle, the allergen is coupled to an insoluble carrier and is allowed to bind the specific IgE antibody which may be present in the serum of a hypersensitive patient. The bound IgE is measured by its reaction with ${ }^{125}$ I-labelled antibody to IgE. For clinical purposes the assay is usually performed with a commercial kit in which the allergens are coupled to paper discs.

The test has been used clinically to show specific $\operatorname{IgE}$ antibody to a variety of allergens including moulds, pollens, dust, insects, and aspergilli. ${ }^{13}$ More recently it has been applied to the diagnosis of allergy to drugs such as rifampicin ${ }^{14}$ and to food antigens such as milk, ${ }^{15}$ eggs, and wheat. ${ }^{16}$ In patients with atopic dermatitis it has been valuable in indicating the responsible allergen. ${ }^{16}$ The correlation between the RAST test on the one hand and the clinical features and skin tests on the other varies in different disorders. With pollens it is good, ${ }^{6} 1718$ but for animal danders and house dust it is much less satisfactory. ${ }^{19}$ Moreover, false-positives are commonly encountered in RAST tests for food allergens, ${ }^{19}{ }^{20}$ and the results may be inappropriate to the extent of the clinical problem-for example, RAST tests may not distinguish patients with atopic dermatitis and allergic rhinitis who develop asthma from those who do not. ${ }^{21} 22$

The standard RAST test suffers from several disadvantages: variable binding properties of IgE antibody in different sera, interference by specific antibody of classes other than $\operatorname{IgE}, 2324$ and the non-standardised units in which the results must necessarily be expressed. ${ }^{5}$ Improved methods include measuring IgE antibody alone, bound to the allergen free from antibodies of other classes, ${ }^{5}$ and the introduction of highly purified antibody to IgE in the final stages of the assay. ${ }^{25}$ Soon enzymelinked reagents are likely to replace radiolabelled materials. Such refinements improve both the specificity and the sensitivity of the RAST technique; but the method, although expensive, is basically safe, easy to perform, and little affected by concurrent treatment.

In contrast, conventional skin tests can be performed simply, cheaply, and without delay but carry the risk of provoking anaphylaxis. ${ }^{13} 26$ Highly purified allergen preparations induce responses that are both sensitive and specific, ${ }^{27}$ but skin testing with reagents in general use produces less useful results. Positive reactions may have little significance: in one survey over $40 \%$ of normal individuals in certain age groups produced reactions to allergens in dusts and moulds, ${ }^{28}$ and clearly most of these were clinically unimportant. Conversely, skin tests may be unexpectedly negative.

Local secretion of specific IgE may predispose to allergic disease in patients in whom the responsible allergen does not elicit positive skin reactions. ${ }^{29}$ Certainly provocation tests may induce a reaction only when the appropriate tissue is challenged -for example, allergic responses in the respiratory tract are 